\title{
Looking at the best indexing method of left atrial volume in the hypertensive setting
}

\author{
Cesare Cuspidi $\mathbb{D}^{1,2} \cdot$ Stefano $^{\text {Carugo }}{ }^{3} \cdot$ Marijana Tadic $^{4}$
}

Received: 30 January 2021 / Accepted: 1 February 2021 / Published online: 11 March 2021

(c) The Japanese Society of Hypertension 2021

Systemic hypertension adversely impacts cardiac structure and function, causing a wide spectrum of alterations, such as myocyte hypertrophy and interstitial fibrosis leading to left ventricular (LV) concentric remodeling, increased LV mass, and systolic and diastolic dysfunction.

Furthermore, an impressive amount of evidence suggests that left atrial enlargement (LAE), identified by assessing the diameter, or more accurately, the volume with transthoracic echocardiography, is a reliable index of a sustained increase in LV filling pressure in the hypertensive setting [1].

LA acts as a volume sensor of the heart, and its dilatation reflects altered dynamics of ventricular relaxation and filling. The close association between LA volume (LAV) and LV diastolic dysfunction has resulted in LAV becoming one of the key diagnostic criteria for LV diastolic dysfunction [2].

LAE is regarded as a strong risk marker for the future development of atrial fibrillation and a robust, independent predictor of heart failure hospitalizations, stroke, and allcause death. The mechanism accounting for the association between LAE and cardiovascular events is multifactorial and may be attributed to underlying pathologies causing LA morphofunctional alterations and elevated LV filling pressure and to arrhythmias (i.e., atrial fibrillation), often a consequence of LAE.

The unfavorable prognostic significance of LAE has been further confirmed and expanded in recent years by new

Cesare Cuspidi

cesare.cuspidi@unimib.it

1 Department of Medicine and Surgery, University of MilanoBicocca, Milano, Italy

2 Istituto Auxologico Italiano, Milano, Italy

3 Department of Clinical Sciences and Community Health, University of Milano and Fondazione Ospedale Maggiore IRCCS Policlinico di Milano, Milano, Italy

4 University Hospital "Dr. Dragisa Misovic - Dedinje" Department of Cardiology, Belgrade, Serbia evidence based on novel echocardiographic techniques (i.e., two- or three-dimensional speckle tracking echocardiography) and magnetic resonance imaging. LAE measured by fourand two-chamber cine cardiac magnetic resonance imaging demonstrated a strong independent association with all-cause mortality in a very large, heterogeneous cohort totaling 10890 patients [3]. Over a median follow-up period of 49 months, mild, moderate, and severe LAE was a significant predictor of death. After adjustment for clinically relevant covariates, moderate $(\mathrm{HR}=1.45,95 \% \mathrm{CI}: 1.1-1.89)$ and severe $(\mathrm{HR}=$ 1.64, 95\% CI: 1.29-2.08) but not mild LAE remained independent predictors of all-cause mortality.

Identification of abnormal cardiac phenotypes (i.e., LV hypertrophy and LAE) by echocardiography has a pivotal role in cardiovascular risk stratification and subsequent therapeutic decision making. Methodological aspects related to this technique may affect the precision of cardiac assessment and the correct classification of patients according to subclinical organ damage. Indeed, the accuracy and precision of quantitative echocardiography are related to multiple factors, including operator experience, patient echogenic characteristics, equipment technology, reliable reading protocols, and appropriate methods for normalizing cardiac parameters for the different body sizes of patients based on precise collection of anthropometric variables. For this latter aspect, it has been reported that indexing echocardiographic parameters to self-reported rather than measured anthropometric values may impair the capacity of detecting LAE. The findings of a multicenter Italian survey showed that misreporting weight and height by individuals attending outpatient echocardiographic laboratories resulted in an underestimation of the prevalence of LAE by $4 \%$ and that misclassification was greater among elderly individuals than among young and middle-aged adults [4].

The value of LAV varies according to sex, age, body size, and ethnicity. The indexing of LAV allows comparison among individuals with different body sizes; however, the best method of normalization for body size remains open to debate. The American Society of Echocardiography 
and the European Association of Cardiovascular Imaging (ASE/EACVI) guidelines recommend indexing the LAV for body surface area (BSA) because the use of this approach also accounts for sex differences in LA size [5].

In this respect, it is worth mentioning that the Normal Reference Ranges for Echocardiography (NORRE) study provided a set of normal echocardiographic values obtained from a cohort of 734 healthy subjects (320 men and 414 women) over a wide range of ages [6]. The study showed significantly different LA sizes and volumes between men and women, but after normalization for BSA, LA volumes were no longer different between groups $\left(27.0 \pm 7.0 \mathrm{ml} / \mathrm{m}^{2}\right.$ in men, $27.3 \pm 7.9 \mathrm{ml} / \mathrm{m}^{2}$ in women). Upper reference values (means $\pm 2 \mathrm{SD}$ ) for LA volumes were $41.9 \mathrm{ml} / \mathrm{m}^{2}$ in men and $41.5 \mathrm{ml} / \mathrm{m}^{2}$ in women using the area-length method. Overall, these data supported the view that for LA volumes, there is a reduced need to check for sexspecific diagnostic cutoff values. In this issue of the journal, the study by Airale et al. [7] adds a new piece of information to the scientific debate related to methods of indexing echocardiographic parameters by specifically focusing on the left atrium in the context of the assessment of hypertension-mediated target organ damage. Before addressing the results of the study, a few aspects of this issue and related topics may warrant some considerations. The Pressioni Monitorate e Loro Associazioni (PAMELA) study showed that LA size exhibited a near-normal distribution in both sexes and the whole population, which means that for several other biological variables, the use of partition values to distinguish abnormality from normality is arbitrary [8]. More importantly, the PAMELA study reported a direct relationship between LA size and many factors (i.e., age, sex, and office, home, and ambulatory BP) and metabolic alterations, which means that its detection should alert the physician to the link between LAE and the presence of cardiovascular risk factors.

A meta-analysis of 15 studies in a pooled hypertensive population of 10,141 patients documented that echocardiographic LAE is frequently detected in treated and untreated hypertensive patients, with an overall prevalence of $\sim 30 \%$ [9]. In a sex-specific analysis based on $>80 \%$ of the total sample, LAE prevalence was found to be nonsignificantly higher in women than in men $(\mathrm{OR}=1.2395 \%$ CI: $0.83-1.83, p=0.30$ ).

In a multicenter study involving 2170 hypertensive individuals (mean age, 62 years; $53 \%$ men) referred by practitioners to 17 outpatient echocardiographic laboratories across Italy, prevalence rates of LAE in both sexes showed different trends depending on the diagnostic criteria used [10]. LAE according to absolute LA diameter was present in $39.5 \%$ of men and $35.5 \%$ of women $(p=n s)$, whereas a significant opposite trend was found by using the value normalized to BSA ( $14.3 \%$ versus $26.9 \%$, respectively; $p<0.01)$. The more pronounced LA remodeling in women was associated with a constellation of risk factors such as older age, higher systolic and pulse pressures, and higher prevalence of obesity and $\mathrm{LVH}$.

In this line of research, the paper by Airale et al. tested the difference in LAE prevalence by using two different diagnostic criteria: (I) an isometric and non-sex-specific criterion (i.e., LA volume $>34 \mathrm{ml} / \mathrm{m}^{2}$ ) endorsed by the ASE/EACVI guidelines, and (II) an allometric and sex-specific criterion (i.e., $>18.5 \mathrm{ml} / \mathrm{h}^{2}$ in males and $>16.5 / \mathrm{h}^{2}$ in females) cited in the 2018 European Hypertension Society/European Cardiology Society (ESH/ESC) guidelines. For this purpose, 441 essential hypertensive patients (mean age, $60 \pm 14$ years; $52 \%$ men; $26 \%$ obese and $10 \%$ diabetic) were enrolled in the study. Approximately one-third of the patients had LVH (24\% men and $32 \%$ women), most of the concentric type. In the whole population, the prevalence of LAE was twice as high when using allometric sex-specific indexation rather than BSA $(50.6 \%$ vs. $23.4 \%, p<0.001)$. The corresponding figures were $42.8 \%$ versus $23.1 \%$ in men and $59.0 \%$ versus $23.6 \%$ in women. According to the absence or presence of LAE, the patients were further classified into three groups: (A) normal LA size ( $n=218$; mean age, $55 \pm 13$ years; $40 \%$ women), (B) LAE defined by allometric indexation ( $n=120$; mean age, $64 \pm 14$ years; $63 \%$ women), and (C) LAE defined by both allometric and isometric indexation ( $n=103$; mean age, $67 \pm 15$ years; $49 \%$ women). The prevalence of $\mathrm{LVH}$ increased progressively from group A (14\%) to group B (34\%) and group C (50\%). Of note, a deterioration of diastolic function parameters, as assessed by the ratio of early (E) peak mitral inflow velocity to mitral (septal and lateral) annular velocity (E/e' ratio), occurred in parallel with the increase in LV structural alterations. Consequently, the findings by Airale et al. suggest that indexing for BSA markedly underestimates the prevalence of LAE because in $>25 \%$ of a large number of untreated and treated hypertensive patients classified as being free of abnormalities in LA size, indexation for height ${ }^{2}$ allowed us to identify LAE, indicating unrecognized target organ damage of adverse prognostic significance and thus leading to the reclassification of these patients as high-risk individuals. Interestingly, the underestimation of the risk of LAE was strongly prevalent in the female sex, thus emphasizing the appropriateness of using different sex-specific cutoffs (as widely accepted for the diagnostic criteria of LVH).

The key argument provided by the authors supporting the clinical value of LAE, identified only by height ${ }^{2}$, relies on its association with LV remodeling expressed by increased LV mass and prevalence of $\mathrm{LVH}$, as well as an impairment in diastolic dysfunction. The strength of this argument is, however, limited by the fact that patients diagnosed with LAE by height ${ }^{2}$ were older ( +9 years) and more likely to be obese and had higher pulse pressure than their counterparts 
with normal LAVs. In fact, these differences, not adjusted in the statistical analysis, may explain per se the greater involvement of subclinical cardiac organ damage in this subset of patients. Regardless of the limits recognized by the authors themselves, this study has the merit of shedding light on a topic that has been neglected so far, represented by the need to use sex-specific diagnostic criteria in phenotyping an essential component of hypertension-mediated organ damage such as LAE. From this perspective, further crosssectional and longitudinal studies are needed, aimed at defining (I) the normal values of the LAV indexed by height ${ }^{2}$ in the context of large cohorts representative of the general population and (II) its independent prognostic value.

\section{Compliance with ethical standards}

Conflict of interest The authors declare no competing interests.

Publisher's note Springer Nature remains neutral with regard to jurisdictional claims in published maps and institutional affiliations.

\section{References}

1. Nwabuo CC, Vasan RS. Pathophysiology of hypertensive heart disease: beyond left ventricular hypertrophy. Curr Hypertens Rep. 2020;22:11.

2. Heitmann KA, Løchen ML, Hopstock LA, Stylidis M, Welde B, Schirmer $\mathrm{H}$, et al. Cross-sectional associations between accelerometry-measured physical activity, left atrial size, and indices of left ventricular diastolic dysfunction: the Troms $\varnothing$ Study. Prev Med Rep. 2020;21:101290.
3. Khan MA, Yang EY, Zhan Y, Judd RM, Chan W, Nabi F, et al. Association of left atrial volume index andall-cause mortality in patients referred for routine cardiovascular magnetic resonance: a multicenter study. J Cardiovasc Magn Reson 2019;21:4.

4. Cuspidi C, Negri F, Muiesan ML, Grandi AM, Lonati L, Ganau A, et al. Working Group on Heart and Hypertension of the Italian Society of Hypertension. Indexing cardiac parameters in echocardiographic practice: do estimates depend on how weight and height have been assessed? A study on left atrial dilatation. J Am Soc Hypertens. 2011;5:177-83.

5. Kou S, Caballero L, Dulgheru R, Voilliot D, De Sousa C, Kacharava G, et al. Echocardiographic reference ranges for normal cardiac chambersize: results from the NORRE study. Eur Heart $\mathbf{J}$ Cardiovasc Imaging. 2014;15:680-90.

6. Lang RM, Badano LP, Mor-Avi V, Afilalo J, Armstrong A, Ernande L, et al. Recommendations for cardiac chamber quantification by echocardiography in adults: an update from the American Society of Echocardiography and the European Association of Cardiovascular Imaging. Eur Heart J Cardiovasc Imaging. 2015;16:233-70.

7. Airale L, Paini A, Ianniello E, Mancusi C, Moreo A, Gaudo G, et al. Left atrial volume indexed for height ${ }^{2}$ is a new sensitive marker for subclinical cardiac organ damage in female hypertensive patients. Hypertens Res. 2021; https://doi.org/10. 1038/s41440-021-00614-4.

8. Bombelli M, Facchetti R, Cuspidi C, Villa P, Dozio D, Brambilla $\mathrm{G}$, et al. Prognostic significance of left atrial enlargement in a general population: results of the PAMELA study. Hypertension 2014;64:1205-11.

9. Cuspidi C, Rescaldani M, Sala C. Prevalence of echocardiographic left-atrial enlargement in hypertension: a systematic review of recent clinical studies. Am J Hypertens. 2013;26:456-64.

10. Cuspidi C, Negri F, Lonati L, Muiesan ML, Capra A, Milan A, et al. Working Group on Heart and Hypertension of the Italian Society of Hypertension. Prevalence and correlates of echocardiographic left atrial enlargement in hypertensive outpatients in clinical practice. Clin Exp Hypertens. 2011;33:328-35. 